\title{
Delivery siRNA with a novel gene vector for glioma therapy by targeting Gli I
}

This article was published in the following Dove Press journal:

International Journal of Nanomedicine

\author{
Peizhi Zhou ${ }^{1, *}$ \\ Yue $\mathrm{Cao}^{2, *}$ \\ Xiaoxiao Liu $^{3}$ \\ Ting $\mathrm{Yu}^{3}$ \\ Qian $X u^{3}$ \\ Chao You' \\ Xiang Gaol,3 \\ Yuquan $\mathrm{Wei}^{2}$ \\ 'Department of Neurosurgery, West \\ China Hospital/West China Medical \\ School, Sichuan University, Chengdu \\ 6I004I, People's Republic of China; \\ 2Department of Pathology, Clinical \\ Medicine College, Chengdu University \\ of Traditional Chinese Medicine, \\ Chengdu, People's Republic of China; \\ ${ }^{3}$ State Key Laboratory of Biotherapy/ \\ Collaborative Innovation Center for \\ Biotherapy, West China Hospital/ \\ West China Medical School, Sichuan \\ University, Chengdu 61004I, People's \\ Republic of China \\ *These authors contributed equally \\ to this work
}

Background: Gene therapy has recently shown considerable clinical benefit in cancer therapy during the past few years, and the application of this choice in cancer treatments is increasing continually. Gli1 is an ideal candidate target for cancer gene therapy and is important for tumorigenesis.

Methods: In this study, we developed a novel gene delivery system with a self-assembly method by using a 1,2-dioleoyl-3-trimethylammonium-propane and methoxy poly (ethylene glycol)-poly(lactide) copolymer (DMP), with zeta potential of $32.7 \mathrm{mV}$ and measuring $35.6 \mathrm{~nm}$. The effect of this delivery system was tested in vitro and in vivo.

Results: DMP showed good performance in delivering siRNA to glioma cells in vitro with high transfection performance (98\%). Moreover, DMP-Gli1si shows a satisfactory antiglioma effect via induction of cell apoptosis and cell growth inhibition in vitro. Furthermore, for subcutaneous tumor-bearing mice, treatment with the DMP-Gli1si complex significantly inhibited tumor growth by inhibiting Gli1 protein expression, promoting apoptosis, and reducing proliferation.

Conclusion: The complex of Gli1 siRNA and DMP may potentially play an important role as a new drug in the clinical treatment of gliomas.

Keywords: glioma, gene therapy, Gli1, MPEG-PLA, DOTAP

\section{Introduction}

Primary malignant tumors of the human brain are the most common causes of cancerrelated death in young adults and children. ${ }^{1}$ Gliomas originate from glial (precursor) cells and have the highest incidence among primary malignant brain tumors. Glioma, which diffusely grows into the brain parenchyma along myelinated axon bundles, is usually progressive and demonstrates relative resistance to both radiotherapy and most chemotherapeutic agents. Surgery and radiotherapy mostly deal with the distinguishable part of the tumor; cells that have diffusely infiltrated into the adjacent brain tissue remain relatively unaffected and give rise to recurrence. Significant progress has recently been made in glioma management based on multidisciplinary treatment. Although the survival time of glioblastoma patients has slightly improved over the past decades, the clinical effects and prognosis are still poor.

Small-molecule-targeted therapy of RNA interference (RNAi) by siRNA holds great promise among various gene therapies for tumors. However, the high delivery efficiency of small RNAs in vivo represents a major challenge in achieving the potency required for clinical application. RNAi is now regaining its momentum after some ups and downs. ${ }^{2}$ Various delivery techniques have been developed for RNAi. Viral vectors can achieve high delivery efficiency and sustained gene silencing with a single injection, offering a practical advantage for disease originating in hard-to-reach
Correspondence: Xiang Gao

Department of Neurosurgery, West China Hospital/West China Medical

School, Sichuan University, No. 37 Gue

Xue Xiang, Chengdu 61004I, People's

Republic of China

Tel +862885422136

$\mathrm{Fax}+862885502796$

Email xiangxianggao2008@163.com
International Journal of Nanomedicine 20 I8: | 3 478|-4793

4781

Dovepress f $y$ in $\mathbf{0}$

http://dx.doi.org/1 $0.2147 /$ IJN.SI 64364 (c) (1) (5) 2018 Zhou et al. This work is published and licensed by Dove Medical Press Limited. The full terms of this license are available at https://www.dovepress.com/terms.php cc) hereby accept the Terms. Non-commercial uses of the work are permitted without any further permission from Dove Medical Press Limited, provided the work is properly attributed. For permission for commercial use of this work, please see paragraphs 4.2 and 5 of our Terms (https://www.dovepress.com/terms.php). 
organs, such as the brain. ${ }^{2,3}$ However, the bio-safety risk restricts its clinical application. Therefore, the development of a safe nonviral vector gene therapy with more efficiency represents a promising therapeutic method. Nonviral vectors, such as cationic lipids and polymers, are developed to improve delivery efficiency and safety. ${ }^{4,5}$ SiRNAs playing roles as candidates for further therapeutic choice have gained more attention. However, therapeutic application of siRNAs depends on its efficient and safe intracellular delivery.

The glioma-associated oncogene homolog 1 (Gli1) was initially named in glioma research, and its product Glil functions as an effector of the Hedgehog (Hh) signaling pathway. ${ }^{6}$ It is a transcriptional factor and may be a potential molecular target for glioma treatment. Glil can affect glioma cell apoptosis and proliferation via regulated cyclin D1 and Bcl-2; moreover, they are key points in signaling pathways of many other tumors and function as effectors for some chemotherapy drugs. ${ }^{8-11}$

In this article, we describe the efficacy of the 1,2-dioleoyl3-trimethylammonium-propane (chloride salt) (DOTAP)conjugated methoxy poly(ethylene glycol)-poly(lactide) copolymer (MPEG-PLA), a nonviral gene delivery vector for delivering a therapeutic gene to human primary glioma cells. Because it displayed affinity, the effect of DMP-Gli1si in a mouse glioma model was tested further.

\section{Materials and methods Materials}

Materials were purchased from standard sources: DOTAP (Avanti Polar Lipids Inc., Alabaster, AL, USA); MTT (MilliporeSigma Co., St. Louis, MO, USA); DMEM and fetal bovine serum (FBS; Thermo Fisher Scientific, Waltham, MA, USA); methanol and acetic acid (high performance liquid chromatography grade; Thermo Fisher Scientific, Waltham, MA, USA); and DMSO and acetone (KeLong Chemicals, Chengdu, Sichuan, People's Republic of China). Antibodies used included rabbit anti-mouse Ki67 antibody and rhodamine-conjugated secondary antibody (both from Abcam, USA).

The MPEG (2000)-PLA(4000) diblock copolymer with a designed molecular weight of 3,000 Da was synthesized through the opening of the L-lactide ring and initiated by MPEG. The MPEG (5.0 g) was melted in a $70-\mathrm{mL}$ flask following the addition of $\mathrm{Sn}(\mathrm{Oct}) 2(1 \mathrm{~mL})$ and anhydrous L-lactide (10 g) under nitrogen. The reactant mixture was maintained for 1 day at $125^{\circ} \mathrm{C}$. The crude product was dissolved in tetrahydrofuran and then purified by precipitation in ice-cold diethyl ether, followed by filtration. Finally, the product was vacuum dried at ambient temperature. The average molecular weight number $(\mathrm{Mn})$ of the MPEG-PLA copolymer was 6,010 Da (data not shown). MPEG (with a molecular weight of 2,000 Da; Sigma-Aldrich Co.) was dried in a one-necked flask under vacuum and stirred at $105^{\circ} \mathrm{C}$ for $90 \mathrm{~min}$ before use.

\section{Preparation of DOTAP/MPEG-PLA}

MPEG-PLA (90 mg) and DOTAP (10 mg) were dissolved in acetone $(2 \mathrm{~mL})$. The mixture was then placed in a roundbottomed flask, and acetone was removed by way of a water bath $\left(55^{\circ} \mathrm{C}\right)$ under negative pressure conditions $(20 \mathrm{~min})$. Finally, a $5 \%$ glucose solution (GS; $5 \mathrm{~mL}$ ) was added to prepare the DMP gene carrier.

\section{Characterization of DMP}

The morphological characteristics of DMP were observed using transmission electron microscopy (TEM; FEI Tecnai $\mathrm{G}^{2}$ F20, Hillsboro, OR, USA). Prior to analysis, samples were diluted with distilled water, placed on a copper grid, stained with molybdophosphoric acid (1 $\mathrm{min}$ ), and allowed to dry at room temperature. Mean particle size and zeta potential of DMP were determined by a Zetasizer NanoZS ZEN 3600 (Malvern Instruments, Ltd., Malvern, Worcestershire, UK).

\section{Agarose gel electrophoresis of naked siRNA and DMP-siRNA complexes}

After DMP-Gli1si was prepared, agarose gel electrophoresis was conducted in $\mathrm{pH}$ 7.4 TAE buffer containing Gold View to stain nucleic acids. Briefly, DMP was mixed with siRNA in increasing ratios $(10: 1,25: 1,50: 1$, and 100:1) to form complexes. Gel retardation assays were conducted on a 1\% agarose gel (Invitrogen Corp., Carlsbad, CA, USA) in tris-acetate running buffer containing Gold View (120 V for $20 \mathrm{~min}$ ). Results were documented using a standard imaging system (BioRad Laboratories, Hercules, CA, USA).

\section{Cell culture and in vitro gene transfer}

The human glioma cell line U87 was purchased from the American Type Culture Collection (ATCC; Manassas, VA, USA) and were cultured using a standard sterile procedure in DMEM (Gibco-BRL, Rockville, IN, USA) containing 10\% FBS (Sigma Chemical Co., St Louis, MO, USA), 100 U/mL penicillin, and $100 \mu \mathrm{g} / \mathrm{mL}$ streptomycin. Cells were maintained with humidity and $5 \% \mathrm{CO}_{2}$ at $37^{\circ} \mathrm{C}$.

U87 cells were seeded on a Costar six-well plate (Corning Inc., Corning, NY, USA) at a density of $4 \times 10^{5}$ cells/ well in a $2 \mathrm{~mL}$ complete DMEM culture medium. After 
$24 \mathrm{~h}$ of incubation, the media was replaced with $800 \mu \mathrm{L}$ serum-free DMEM per well. DMP/Gli1siRNA (siGli1-1: GCCTGAATCTGTGTATGAA; siGli1-2: GGACAGAA CTTTGATCCTT), DMP-Consi, or DMP in a final volume of $200 \mu \mathrm{L}$ were subsequently added to designated wells and allowed to incubate for $5 \mathrm{~h}$ before replacement with DMEM. Fluorescein isothiocyanate (FITC)-siRNA-transfected cells were observed under a fluorescence microscope (Olympus IX73, U-HPLGPS, Olympus Corporation, Shinjuku, Tokyo, Japan) following an additional 24-h incubation, and transfection efficiency was determined via FACS flow cytometry (BD Biosciences, San Jose, CA, USA).

\section{MTT assay}

U87 cells were seeded on 96-well plates (Corning Inc.) at a density of 5,000 cells per well with DMEM. After 24 h, U87 cells were transfected with DMP, DMP-Consi, or DMPGli1si, or treated with GS as a negative control. After $72 \mathrm{~h}$, $20 \mu \mathrm{L}$ MTT was added to each well, and the cells were further cultured at $37^{\circ} \mathrm{C}$ for an additional $4 \mathrm{~h}$. The surviving cells converted MTT to formazan, which generates a purplish blue color when dissolved in DMSO. The color intensity was measured at $570 \mathrm{~nm}$ using a plate reader (OPTImax, Molecular Dynamics).

\section{Cell apoptosis assay}

U87 cells were seeded on six-well plates (Corning Inc.) at a density of $3 \times 10^{5}$ cells per well with DMEM. After 24 h, U87 cells were transfected with DMP, DMP-Consi, or DMP-Gli1si or treated with GS as a negative control. After $72 \mathrm{~h}$, the extent of apoptosis in U87 was evaluated by flow cytometric analysis (FCM; BD FACSCalibur, USA) using FITC-conjugated Annexin V/propidium iodide (PI; BD PharMingen) staining according to the manufacturer's instructions. Both early apoptotic (Annexin V-positive and PI-negative) and late apoptotic (Annexin V-positive and PI-positive) cells were detected by FCM.

\section{Western blot}

U87 cells were seeded on $10 \mathrm{~cm}$ plates (Corning Inc.) at a density of $3 \times 10^{6}$ cells per well with DMEM. After $24 \mathrm{~h}, \mathrm{U} 87$ cells were transfected with DMP-Consi or DMP-Gli1si, and DMP as a negative control. After $72 \mathrm{~h}$, cells were washed with cold PBS and lysed with a buffer containing $20 \mathrm{mM}$ Tris$\mathrm{HCl}$ ( $\mathrm{pH} 7.5$ ), $150 \mathrm{mM} \mathrm{NaCl}, 1 \mathrm{mM}$ EDTA, and 1\% Triton $\mathrm{X}-100$ in the presence of protease inhibitors. Total protein concentration was measured using the BCA protein assay kit (Thermo Scientific). The protein extracts were separated by sodium dodecyl sulfate-polyacrylamide gel electrophoresis and transferred to polyvinylidene fluoride membranes (BioRad). The membranes were then probed with primary antibody against Bax, Bcl-2, MAPK, p-MAPK, and $\beta$-actin, followed by incubation with secondary antibodies. The blots were visualized by an enhanced chemiluminescence detection system (Amersham Biosciences Corp., Piscataway, NJ, USA). This process was repeated in triplicate.

\section{Glioma mouse model establishment}

U87 glioma cells were obtained from the ATCC, cultured in DMEM supplemented with $10 \% \mathrm{FBS}$, and maintained at $37^{\circ} \mathrm{C}$ with $5 \% \mathrm{CO}_{2}$. All animal experiments were conducted in accordance with the guidelines of the Animal Care Committee of Sichuan University (Chengdu, People's Republic of China) and were approved by the Animal Care Committee of Sichuan University. Female BALB/c nude mice (6-8 weeks old) were purchased from Vital River (Beijing, People's Republic of China) and housed in a specific pathogen-free (SPF) environment at stable room temperature and humidity and were handled in strict accordance with proper, standard animal practices.

In vivo mouse models of glioma were established by subcutaneous (s.c.) injection of U87 cells $\left(1 \times 10^{7}\right.$ cells in $0.2 \mathrm{~mL}$ serum-free DMEM). Mice were randomly allocated into four groups (GS, DMP, DMP-Consi, or DMP-Gli1si). To assess tumor growth, treatment began 7 days (in the s.c. model) after inoculation. Mice in the experimental group (DMP-Gli1si) were given intratumor injections of liposomal siRNA $(1 \mathrm{~nm})$ in $100 \mu \mathrm{L}$ GS every 2 days for 13 total injections, whereas the other groups received the same amount of DMP or DMP-consi in the same volume of GS or received GS only as a negative control.

\section{Proliferation and apoptosis assays of tumor tissues}

Tumor tissue proliferation was immunohistochemically analyzed using a rabbit anti-human Ki67 antibody (Novus Biologicals, Littleton, CO, USA) with a streptavidin-biotin detection method. Ki67 expression was quantified by counting the number of positive cells in 10 randomly selected fields at $200 \times$ magnification. Tumor apoptotic levels were determined using a terminal deoxynucleotidyl transferasemediated nick end labeling (TUNEL) immunofluorescence kit (Promega, Madison, WI, USA) according to the manufacturer's instructions. All sections were observed and digitally photographed under a DM 2500 fluorescence microscope (Leica Microsystems CMS GmbH, Wetzlar, Germany). All of these sections were observed or counted by two investigators or pathologists in a blinded fashion. 


\section{Statistical analysis}

All data were analyzed using GraphPad Prism software (GraphPad, San Diego, CA, USA). Data from multiple groups were analyzed using analysis of variance (ANOVA), and multiple comparisons between the groups were conducted using the Newman-Keuls method after ANOVA. Survival data were plotted using Kaplan-Meier curves and analyzed using the log-rank test. Tumor volumes were calculated as $\left(\right.$ smaller diameter) ${ }^{2}$ (larger diameter) $\times 0.52$. Statistical significance of observed differences in tumor volume and vessel density were analyzed using the Student's $t$-test. All values were presented as the mean \pm the standard error of measurement. A $p$-value of $<0.05$ was considered to be statistically significant for all experiments.

\section{Results}

\section{Preparation and characterization of DMP-siRNA}

DMP and DMP-siRNA were produced by the described self-assembly method (Figure 1A). TEM images depict the bilayer spheroidal morphology of DMP, with an estimated size of $\sim 25 \mathrm{~nm}$ (Figure 1B). Moreover, the zeta potential and size were found to be $36 \mathrm{mV}$ and $37.47 \mathrm{~nm}$ for DMP (Figure 1C and D).

\section{A}
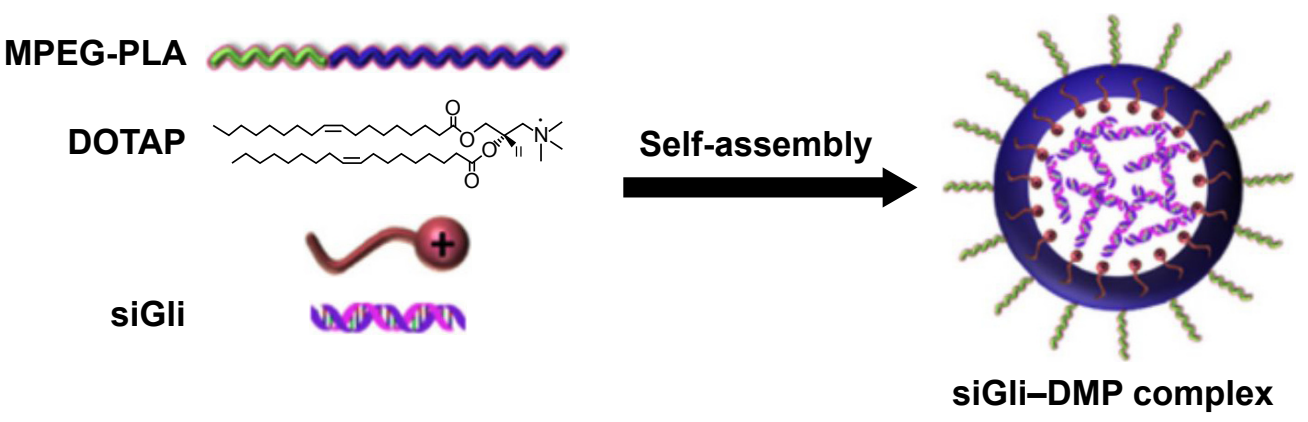

B
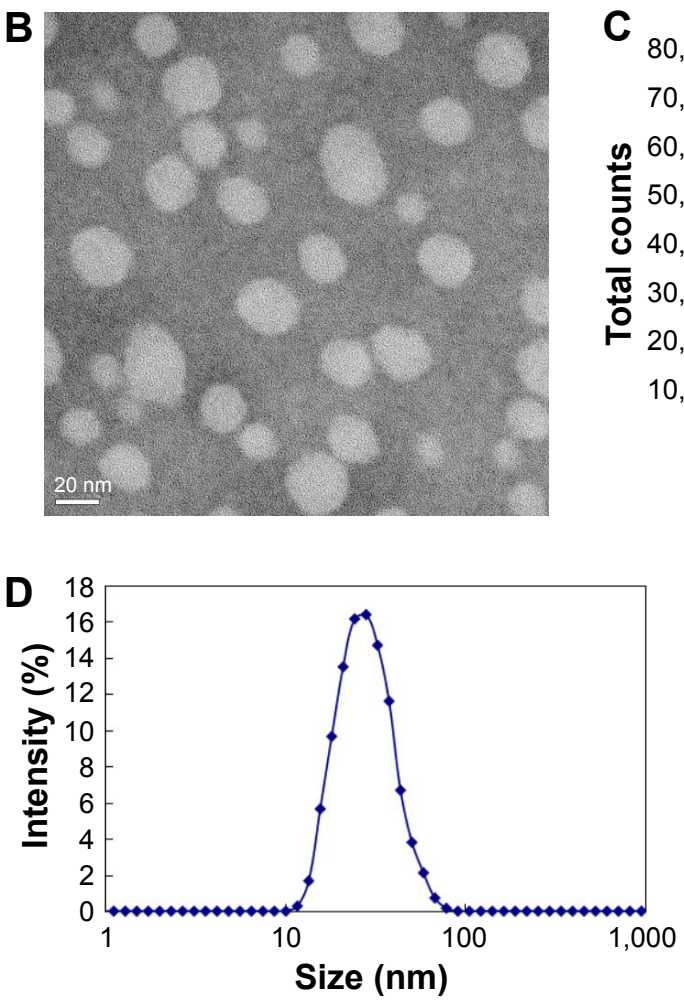
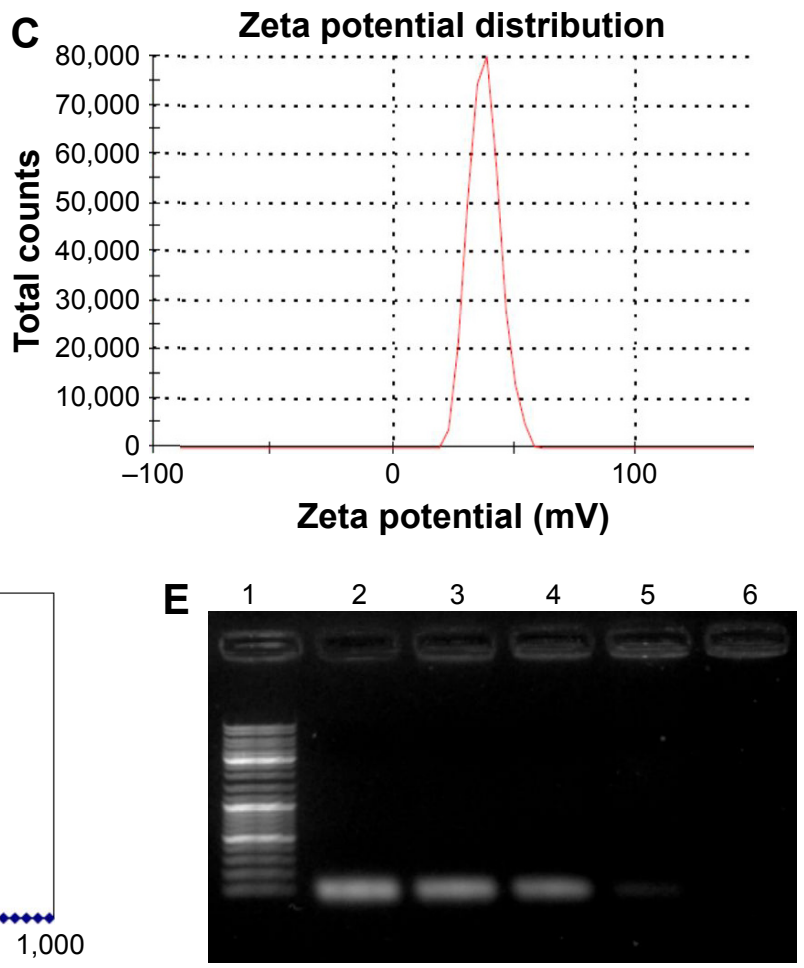

Figure I Preparation and characterization of DMP.

Notes: (A) Preparation of the DMP and Gli lsi complex: first, a novel gene carrier was prepared with a self-assembly method. MPEG-PLA and DOTAP were assembled into a new gene carrier - DOTAP/MPEG-PLA micelles (DMP). Then Glil siRNA was transported into cancer cells by DMP; (B) particle size of DMP; (C) zeta potential of DMP; (D) morphological characteristics of DMP by TEM observation; (E) gel retardation assay of siRNA and complexes. Lane I, DNA marker; lane 2, naked siRNA; lanes 3-6, different weight ratios of siRNA with DMP (I:10; I:25; I:50, and I:100). siRNA was completely incorporated into DMP at a weight ratio of I:50 and complexes were prepared without free siRNA.

Abbreviations: DOTAP, I,2-dioleoyl-3-trimethylammonium-propane (chloride salt); MPEG-PLA, methoxy poly(ethylene glycol)-poly(lactide) copolymer; DMP, I,2-dioleoyl3-trimethylammonium-propane and methoxy poly(ethylene glycol)-poly(lactide) copolymer; TEM, transmission electron microscopy. 
Gel retardation assays were used to characterize the ability of the DMP liposome to carry siRNA. As shown in Figure 1E, the free siRNA not entrapped in the liposome appear as a bright band (lanes 2-4), and no other band of free RNA was observed in lanes 5-6. This suggests that siRNA was completely incorporated into the DMP and the lipoplexes were prepared successfully without free siRNA at a ratio of 50:1 for DMP:siRNA.

\section{High transfection efficiency of DMP- siRNA in U87 cells}

In the in vitro cell transfection assay, DMP produces high transfection efficiency in U87 cells after incubation for $4 \mathrm{~h}$, as shown in Figure 2A and B. The specific transfection rate demonstrated by FCM was $94.6 \%$ and $98.4 \%$ and DMP were mixed with siRNA-FITC at a ratio of 50:1 and 100:1 after $4 \mathrm{~h}$ of transfection (Figure $2 \mathrm{C}$ and D).
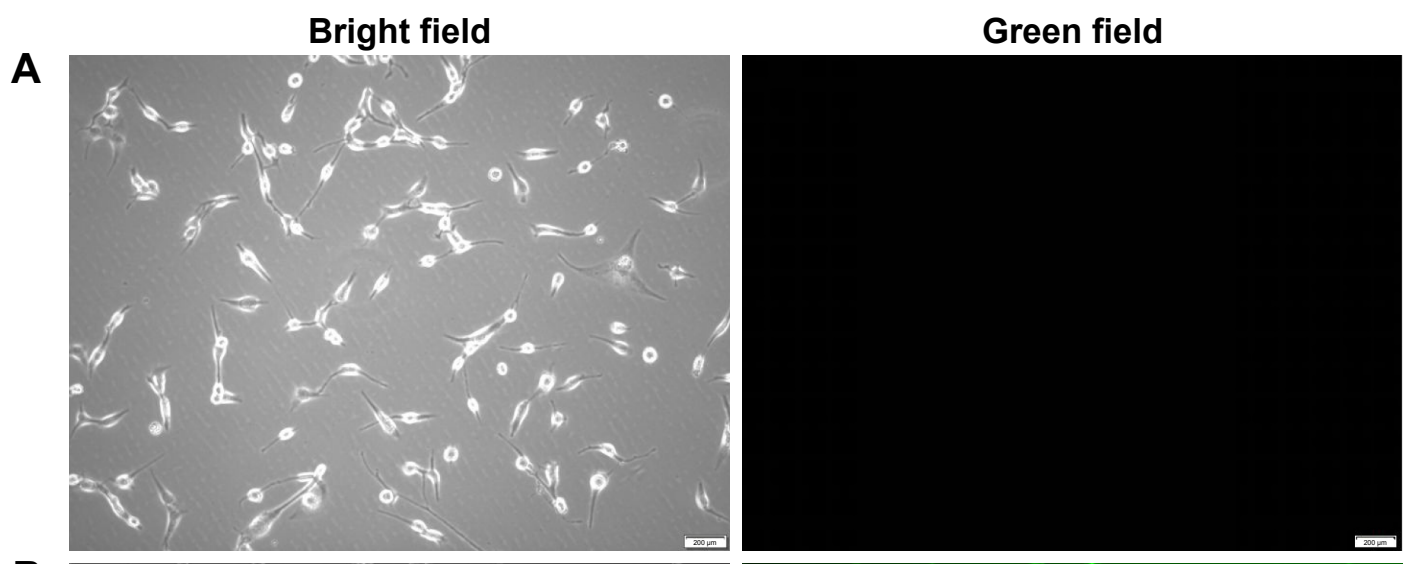

B
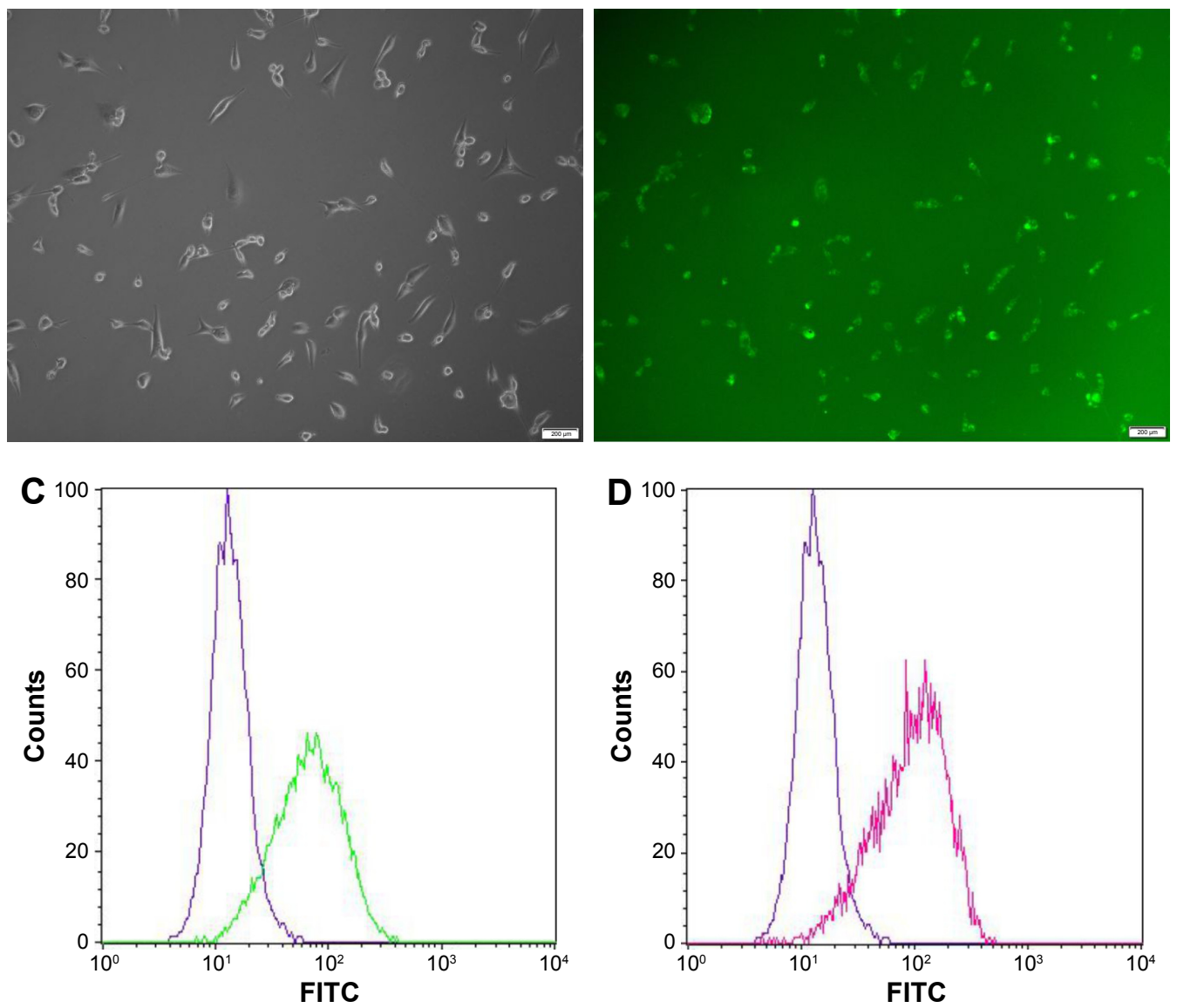

Figure 2 Transfection efficiency measurement of DMP.

Notes: DMP, containing $0.1 \mathrm{~nm}$ siRNA, was used to transfect U87 cells for $4 \mathrm{~h}$. The transfection efficiencies at both weight ratio (DMP [as control A] siRNA vs DMP as $\mathrm{I}: 50$ and $\mathrm{I}: \mathrm{I} 00$ [B]) were determined using fluorescence microscope and flow cytometry (C and D).

Abbreviations: DMP, I,2-dioleoyl-3-trimethylammonium-propane and methoxy poly(ethylene glycol)-poly(lactide) copolymer; FITC, fluorescein isothiocyanate. 
U87 cells were transfected with DMP, DMP-Consi, or DMP-Gli1si or were treated with GS as a negative control. In the DMP-Gli1si group, we found that the expression of Gli1 was significantly lower than in the control groups after $72 \mathrm{~h}$ of incubation in culture (Figure $3 \mathrm{~A}$ ).

\section{In vivo antitumor effect of DMP-Gli Isi} DMP-Glilsi inhibited the proliferation of glioma cell

U87 cells were transfected with DMP_Gli1si, DMP-Consi, or DMP, as a negative control, for $72 \mathrm{~h}$. The MTT result showed that the cell proliferation of glioma cell in the DMPGli1si group was lower than in all control groups $(p<0.05$; Figure 3B).

\section{DMP-Glilsi induced apoptosis of glioma cells}

U87 cells were transfected with DMP-Gli1si, DMP-Consi, or DMP, as a negative control, for $72 \mathrm{~h}$. We then analyzed the potential reason behind growth inhibition of the U87 glioma cells and found evidence that the level of apoptosis in the DMP-Gli1si group was $24.5 \%$ higher than in all the control groups ( $p<0.05$; Figure 4$)$.
Anti-glioma molecular mechanism of DMP-Glilsi in vitro

To investigate the mechanism of the anticancer effect of DMP-Gli1si, protein expressions related to cell apoptosis and proliferation following treatment of DMP-Gli1si, DMP-Consi, and DMP were examined. As demonstrated in Figure 5, the Bcl-2 protein expression was decreased and the Bax expression increased after DMP-Gli1si treatment, indicating that DMP-Gli1si induced apoptosis through the mitochondrial pathway. Finally, the levels of MAPK and p-MAPK after DMP-Gli1si treatment were also tested, and the result showed that p-MAPK was obviously decreased after 72-h treatment with DMP-Gli1si, whereas the total MAPK were unchanged.

\section{In vivo antitumor effect of DMP-Gli Isi}

DMP loaded with con-siRNA and Gli1-siRNA was applied to treat U87 glioma cells in nude Balb/c mice model via intratumor injections. DMP-Gli1si treatment showed a dramatic antitumor effect compared with other lipoplexes (DMP-consi, DMP, and GS); otherwise, no significant differences were observed among the DMP-consi-treated, DMP-treated, and

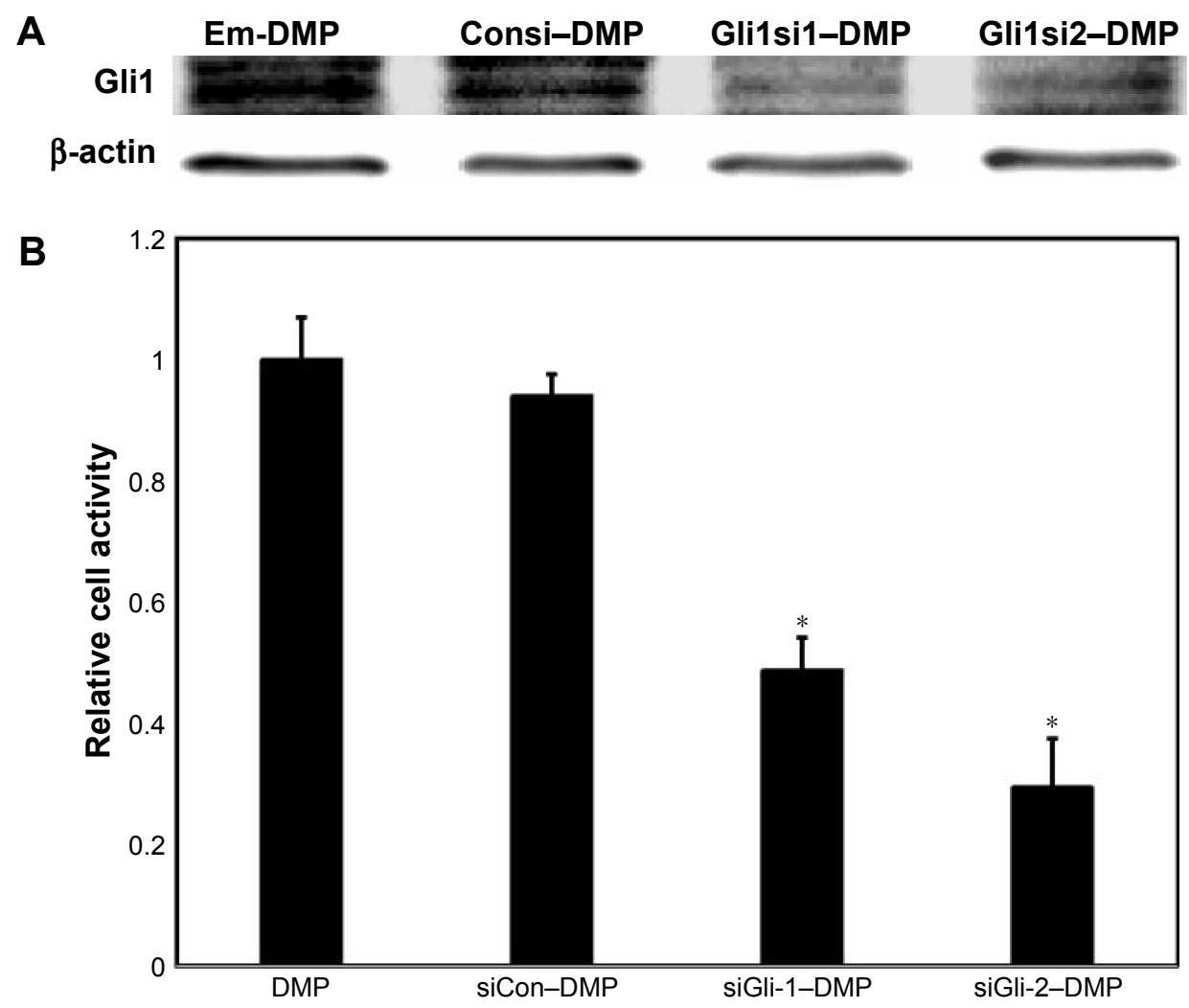

Figure $3 \mathrm{Glil}$ expression detection and MTT test.

Notes: When U87 cells were transfected with DMP, DMP-Consi, or DMP-Gli Isi, Gli I expression was tested by Western bloting (A), and cell activity was tested by the MTT test (B), ${ }^{*} p<0.05$, DMP-Glilsi vs NS and DMP-Consi.

Abbreviations: DMP, I,2-dioleoyl-3-trimethylammonium-propane and methoxy poly(ethylene glycol)-poly(lactide) copolymer; NS, normal salt. 
A

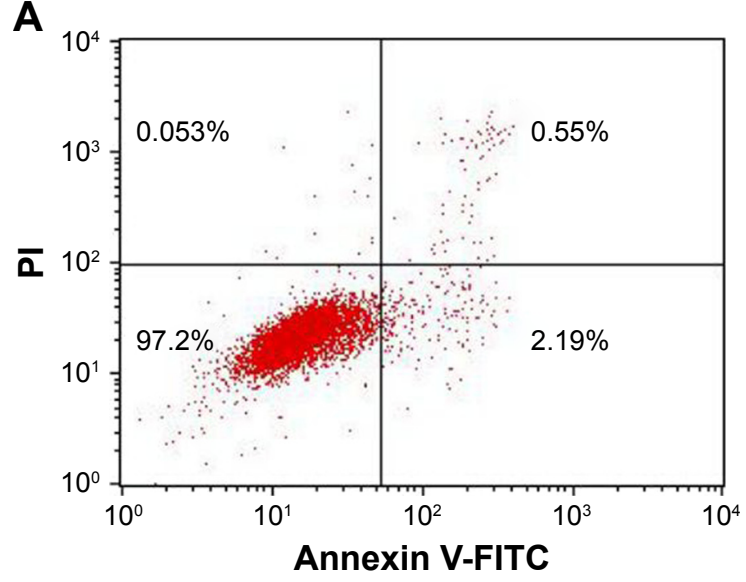

C

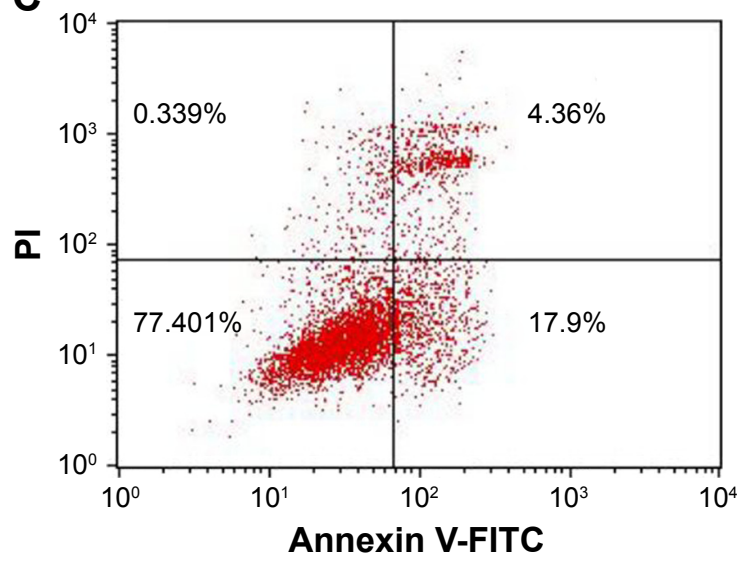

B

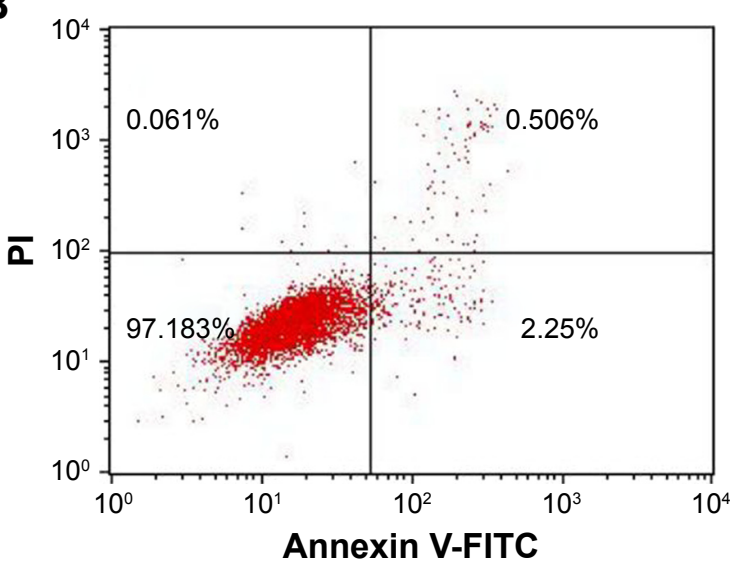

D

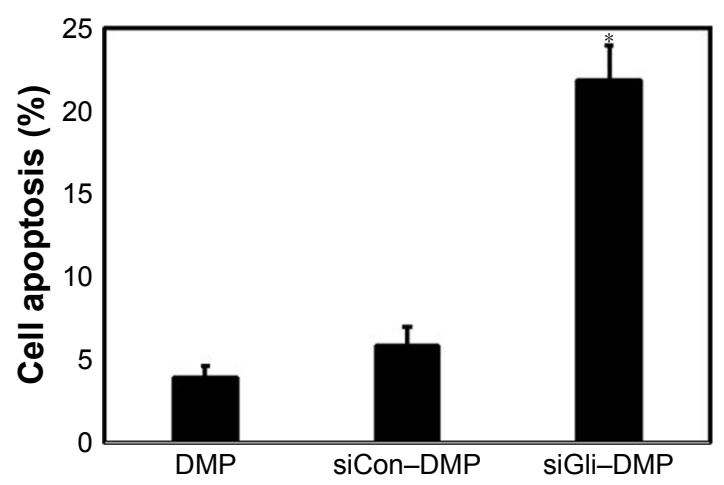

Figure 4 Cell apoptosis test.

Notes: When U87 cells were transfected with DMP (A), DMP-Consi (B), or DMP-Gli Isi (C) for 72 h, U87 cells with different treatment were stained with Annexin V-FITC/PI and tested by flow cytometry. (D) Mean apoptosis cells of different groups. ( ${ }^{*} p<0.05$, DMP-Glil si versus NS A), DMP-Consi (C).

Abbreviations: DMP, I,2-dioleoyl-3-trimethylammonium-propane and methoxy poly(ethylene glycol)-poly(lactide) copolymer; PI, propidium iodide; NS, normal salt.

DMP

$\mathrm{Bcl}-2$

Bax

p-MAPK

T-MAPK
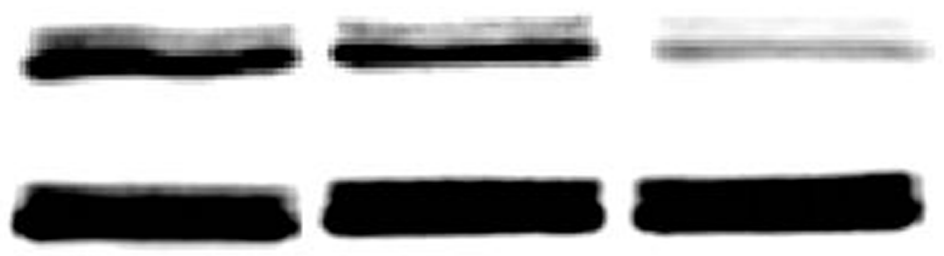

$\beta$-actin

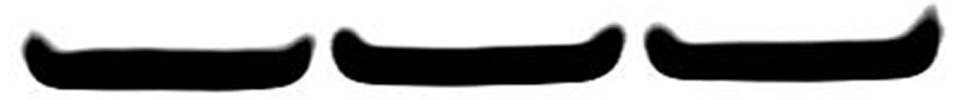

Figure 5 Anti-glioma molecular mechanism of DMP-Gli Isi.

Notes: When U87 cells were transfected with DMP, DMP-Consi, or DMP-Glilsi, the Bcl-2, Bax, p-MAPK, and T-MAPK expressions were tested by Western blotting. Moreover, results showed that levels of $\mathrm{Bcl}-2$ and P-MAPK were downregulated and Bax was upregulated after treatment with DMP-Glilsi.

Abbreviation: DMP, I,2-dioleoyl-3-trimethylammonium-propane and methoxy poly(ethylene glycol)-poly(lactide) copolymer. 

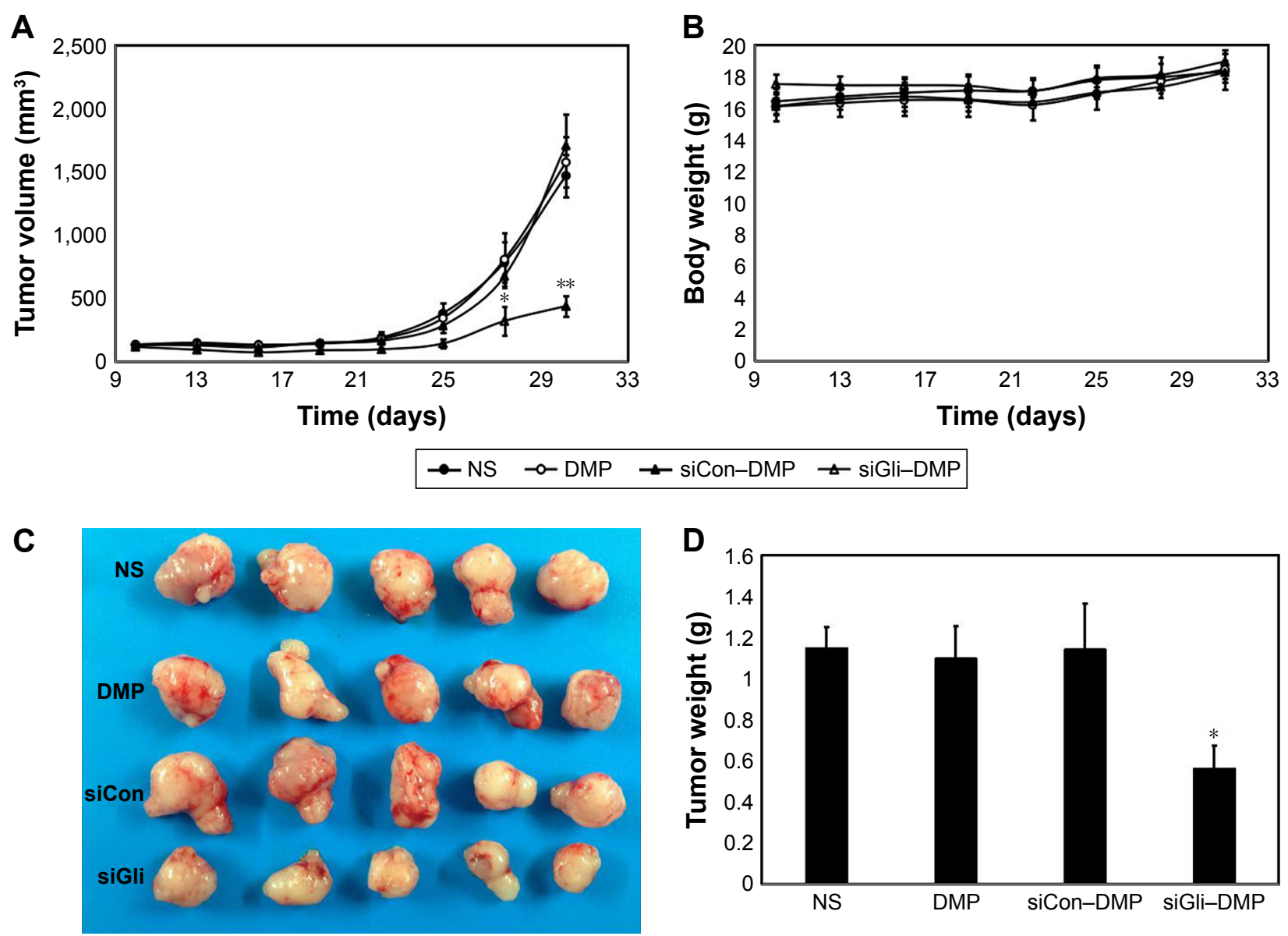

Figure 6 Anti-glioma effect of DMP-Glilsi in vivo.

Notes: (A) Tumor growth curves, (B) body weight curves of different groups, (C) tumor photos of normal salt (NS), DMP, DMP-Consi, and DMP-Gli si treatment groups, and (D) tumor weight of different groups (mean \pm SEM, $n=5)\left({ }^{*} p<0.05\right.$, DMP-Glilsi vs NS, DMP, and DMP-Consi; **p $\left.<0.001\right)$.

control groups. DMP-consi significantly reduces the mass of tumors (with average tumor mass at $0.52 \mathrm{~g}$ ) compared with the DMP (1.24 g), DMP-consi (1.18 g), and GS (1.197 g) groups. In addition, mice treated with DMP-Gli1si were found to have the lowest body weight compared with controls (Figure 6). Furthermore, we detected the expressions of Gli1 in different groups, and results showed that the Gli1 was successfully knocked down after DMP-Gli1si treatment (Figure 7).

\section{DMP-Glilsi induces cancer cell apoptosis and inhibits tumor cell proliferation}

Tumor growth is balanced between apoptosis and proliferation. To explore whether phenotypic changes occurred in the tumor tissues, the percentage of apoptotic and proliferating cells were tested (Figures 8 and 9). The TUNEL assay was used to determine the severity of apoptosis. This assay functions by detecting early DNA fragmentation and allowed us to understand whether apoptosis contributes to the antitumor effects of treatment with DMP-Gli1si observed in vivo.
In the DMP-Gli1si-treated group, 67.5\% TUNEL-positive cells were identified, exceeding that of the DMP-Consitreated group. To assess levels of tumor cell proliferation, Ki67 staining was undertaken. The results showed fewer proliferating cells in tumor tissues treated with DMP-Gli1si than in those treated with DMP-Consi, DMP, or GS. Taken together, this evidence suggests that DMP-Gli1si can successfully deliver Gli1si to tumor cells and generate antitumor effects by activating apoptotic mechanisms and inhibiting tumor cell proliferation.

\section{Discussion}

Cancer is a multifactorial disease and most types are associated with poor prognosis; it is one of the leading causes of human death worldwide. It results from genetic alterations of normal cells and can lead to abnormal proliferation, metastasis, and tumor immunogenicity. The classic treatments for cancer are surgery, radiotherapy, and chemotherapy. Surgery can be used to remove the cancerous tissue from the 

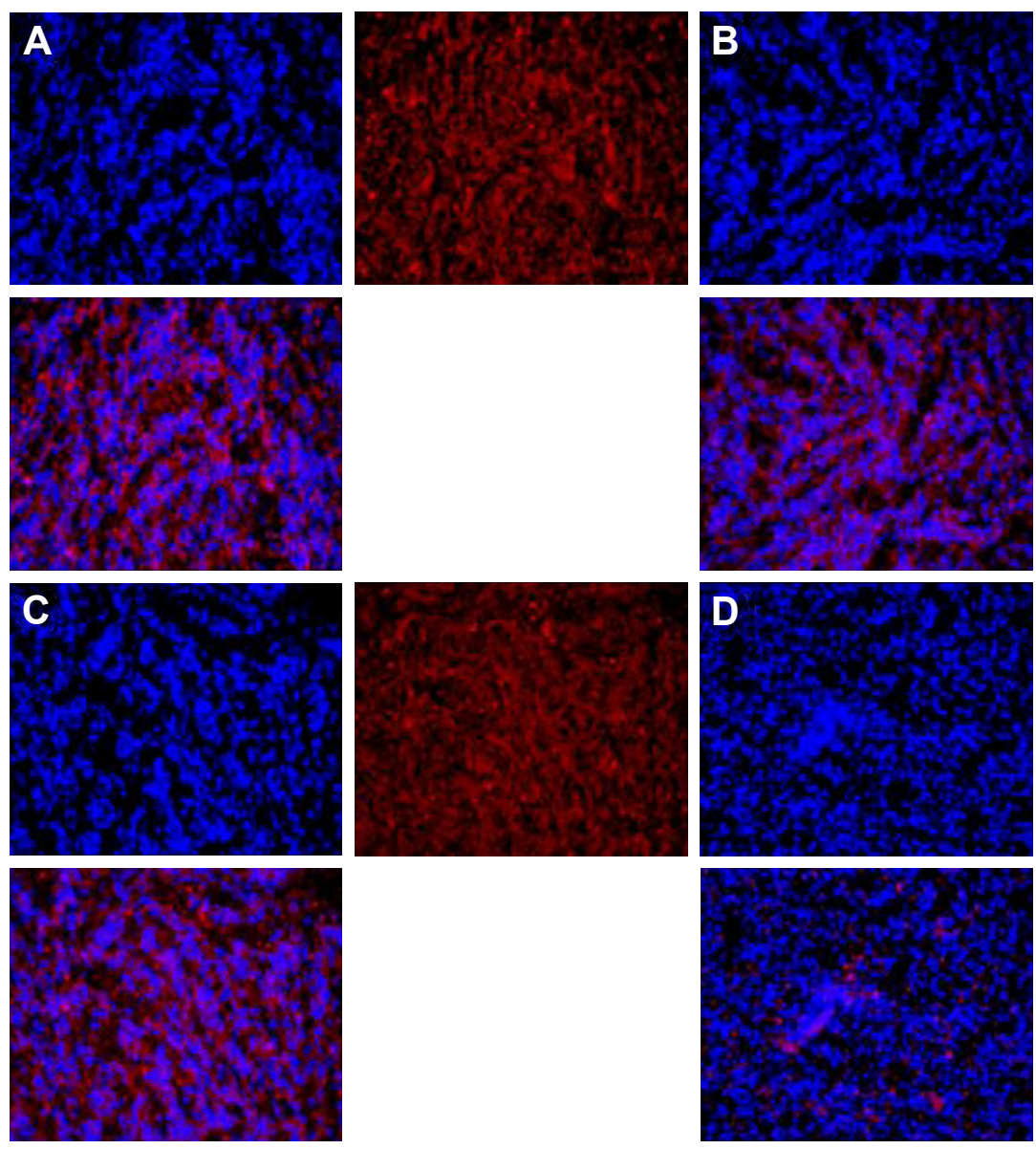
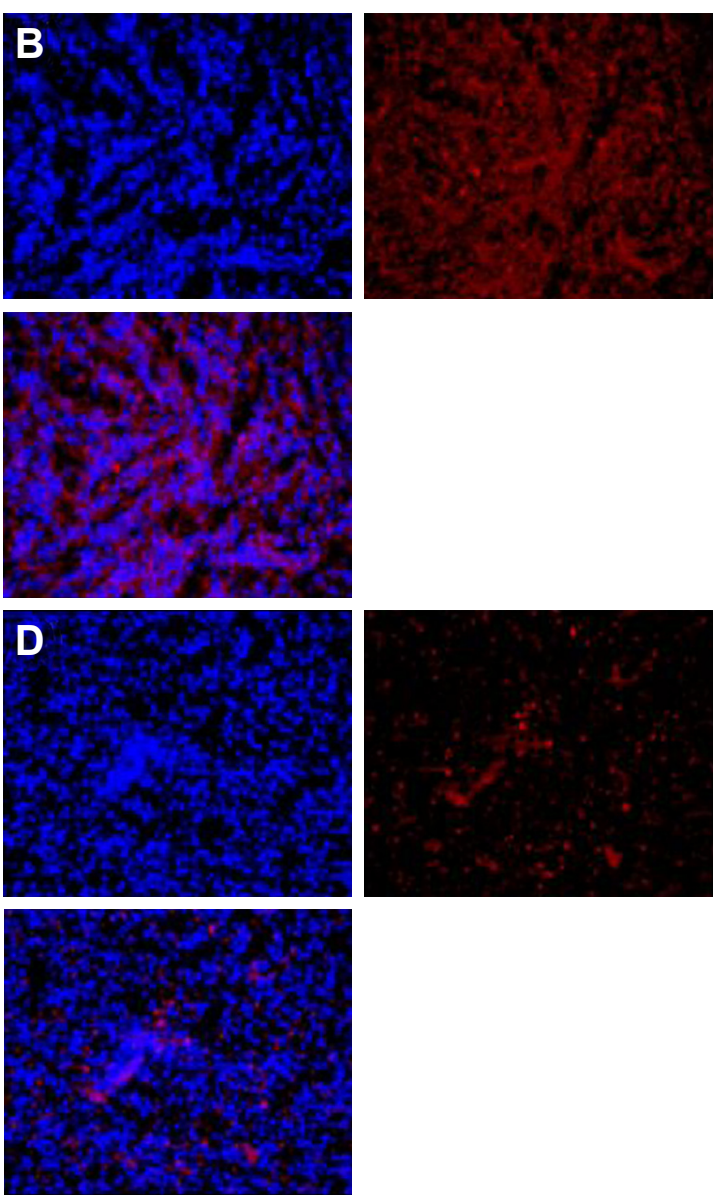

Figure $7 \mathrm{Glil}$ expression in vivo.

Notes: The Glil expression was determined by immunofluorescence with Glil antibody. The Glil expression of NS (A), DMP (B), and DMP-Consi (C) were higher than DMP-Glilsi (D). The result indicated that the Glil was successfully knocked down by the DMP-Glil si complex.

Abbreviations: DMP, I,2-dioleoyl-3-trimethylammonium-propane and methoxy poly(ethylene glycol)-poly(lactide) copolymer; NS, normal salt.

body and get tumor tissue for histopathologic examination. Radiotherapy can injury the DNA and kill cancer cells. Chemotherapy remains the preferred method due to its effectiveness, but its success was hampered as normal cells may also be killed due to the lack of selectivity. Carcinoma cells can acquire a chemoresistant phenotype and induce tumor recurrence. This chemoresistance may be the result of genetic alterations within the cancer cells. For the past few years, gene therapy, which targets specific molecular to treat cancer on the basis of its characteristics, has recently shown considerable clinical benefits in cancer therapy.

Sonic hedgehog (Shh) was first identified in the fruitfly and has a crucial role in development, acting as a morphogen involved it patterning several systems including the vertebrate neural tube. Zinc-finger proteins of the Gli family of transcription are mediators of the Shh signal and can pattern the dorsal-ventral axis of the spinal cord. ${ }^{12}$ Gli1 is one of the three Gli family members and was found to be the most significant target for cancer therapy among the three GLIs; it was initially named in glioma research. Previous research showed that the Gli1 variant is expressed in most glioma tissues and patient-derived xenograft tumor models ${ }^{13}$ but is undetectable in normal brain tissues. Many studies have shown that Gli1 regulates the transcription of various genes directly and are known to be involved in tumor cell survival, proliferation, and chemotolerance. ${ }^{14-16}$ Gli1 could be an important potential target for treating such tumors based on the previous research. More attention and analysis should be undertaken on the roles of the different Glil mutations in the development and progression of cancer.

Gene therapy is a prospective strategy to modulate gene expression in targeted cells to treat human cancers. RNAi is a gene regulation mechanism initiated by different kinds of RNA molecules that enables sequence-specific gene silencing by promoting specific mRNA degradation. Use the specific siRNA as a molecular therapy method has shown great therapeutic potential for abnormal gene overexpression or mutation-based diseases. ${ }^{17}$ 

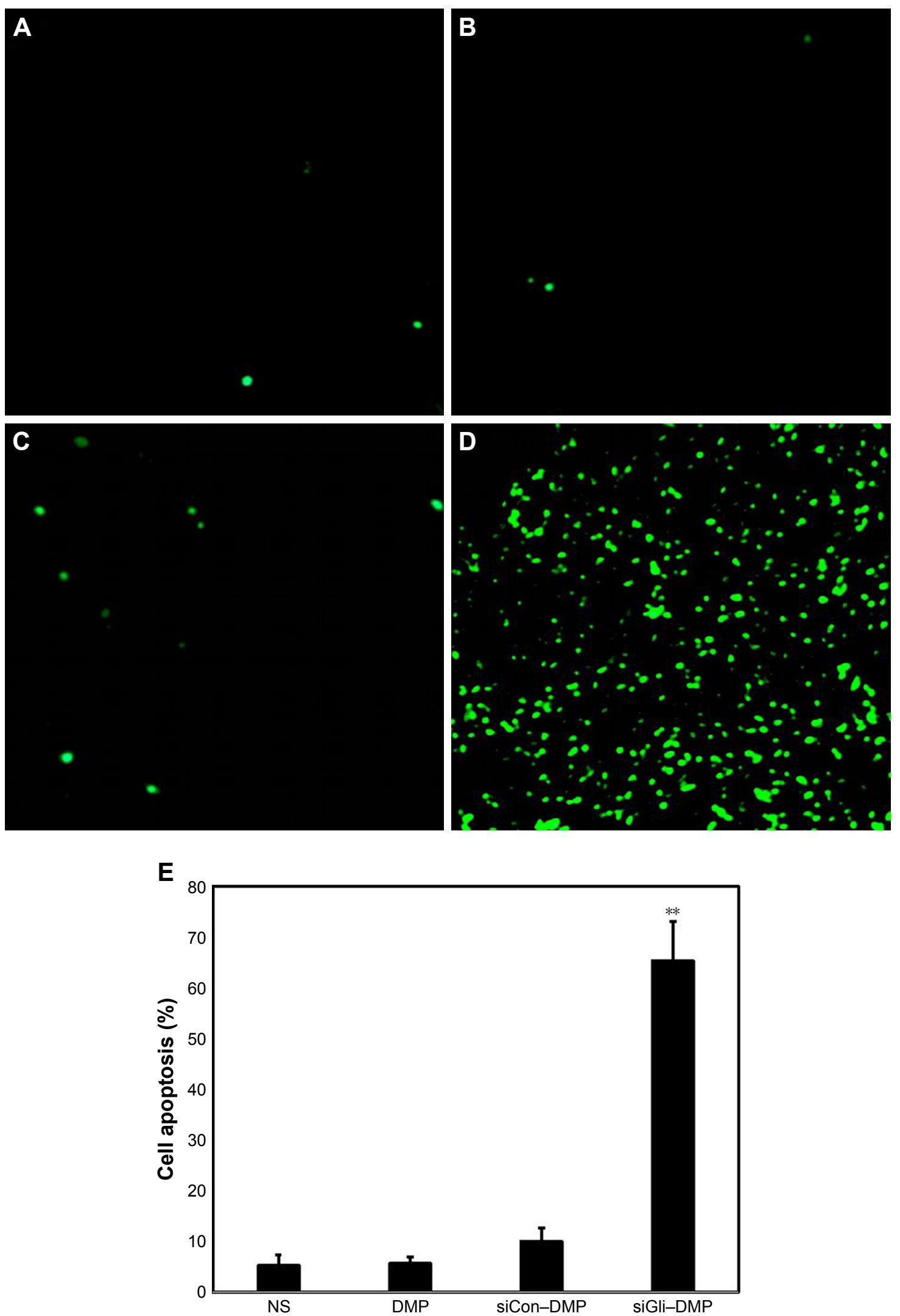

Figure 8 Cell apoptosis detection.

Notes: Cell apoptosis was assessed by counting the number of Tunnel-positive cells in the field (five high-power fields per slide), and DMP-Gli Isi (D) was superior to other controls in increasing cell apoptosis. DMP-Glilsi significantly increased apoptosis (**p<0.00I, DMP-Glilsi vs NS [A], DMP (B), DMP-Consi [C]), and (E) mean apoptosis cells every five fields.

Abbreviations: DMP, I,2-dioleoyl-3-trimethylammonium-propane and methoxy poly(ethylene glycol)-poly(lactide) copolymer; NS, normal salt. 



Figure 9 Cell proliferation detection.

Notes: Cell proliferation was assessed by counting the number of Ki67-positive cells in the field (five high-power fields per slide), and DMP-Glilsi was superior to other controls in inhibiting cell proliferation. DMP-Gli lsi significantly inhibited cell proliferation, (E) mean Ki67-positive cells every five fields; * $<<0.05$, DMP-Gli l si (D) vs NS (A), DMP (B), DMP-Consi (C).

Abbreviations: DMP, I,2-dioleoyl-3-trimethylammonium-propane and methoxy poly(ethylene glycol)-poly(lactide) copolymer; NS, normal salt. 
However, major challenges of siRNA therapeutics involve the effective and stable delivery of siRNA in vivo. Delivery systems of siRNA can be categorized into physical, conjugation, bio-carrier (viruses and bacteria), and nonviral carrier methods. ${ }^{18}$ DNA-based short hairpin RNA (shRNA) expression cassettes are usually delivered to target cells ex vivo via viruses and bacteria; then, these modified cells are reinfused into the patient. ${ }^{19}$ The adenovirus and adenoassociated-virus derived vectors provide efficient delivery for shRNA expression ${ }^{20}$ but may induce insertional mutagenesis and immunogenicity. ${ }^{21}$ The nanotechnological technique has significant advances in developing nonviral gene delivery systems providing safer, easy-to-produce, efficient siRNA delivery systems. SiRNA nanoparticles provide a good potential therapeutic option for cancer therapy with its improved biocompatibility. ${ }^{22}$ In our present study, siRNA strategy was utilized in order to study the potential effect of Gli1 on glioma cells in vitro. Three distinct Gli1-siRNAs with nonoverlapping sequences were introduced to glioma cells, and one of those three applied Gli1-siRNAs dramatically downregulated Gli1mRNA expression in glioma cells. Then, the Gli1-siRNA was encapsulated within nanoparticles.

In this study, we developed a novel gene delivery system with a self-assembly method by MPEG-PLA and DOTAP (DMP) with zeta potential of $26 \mathrm{mV}$ and measuring $50 \mathrm{~nm}$. The siRNA was completely incorporated into the DMP and the lipoplexes were prepared successfully without free siRNA at a ratio of 100:1 of DMP:siRNA. DMP showed good performance with transfection efficiency as high $95 \%$ in delivering siRNA to glioma cells in vitro. The Western blotting assay was used to confirm the knockdown efficiency. The results showing Glil protein expression were significantly reduced by the combination of DMP and siRNA-Gli1.

The Gli1 isoforms have been shown to be involved in the proliferation and apoptosis of cells. For example, ectopic Gli1 expression in embryonic mouse brain leads to neural tissue outgrowth. ${ }^{23}$ Cell proliferation and tumor size could be reduced via inhibition of Shh-Gli1 signaling by Gli1-targeted cyclopamine or siRNA. ${ }^{24}$ Gli1 expression in mammary glands leads to hyperplasia and tumor formation, ${ }^{25}$ whereas knockdown of Gli1 decreases proliferation and enhances apoptosis in metastatic breast cancer cells. ${ }^{26}$ In our present study, we found that the treatment with the combination of siRNA Gli1 and DMP significantly inhibits cell proliferation and promotes cell apoptosis in glioma, possibly by the downregulation of Bcl-2 and upregulation of Bax.

What is more, the DMP-Gli1si complex has significantly inhibited tumor growth in the subcutaneous tumor-bearing mice model in vivo by inhibiting Gli1 protein expression, promoting apoptosis, and reducing proliferation.

In conclusion, our study demonstrates that the combination of siRNAGlil and DMP is more effective in inhibiting the proliferation of glioma cells as well as providing a theoretical basis and part of technical methods for the treatment of gliomas with the combination of molecular targeted therapy.

\section{Acknowledgments}

This work was supported by the National Natural Science Foundation of China (NSFC81502165) and the Sichuan University Outstanding Young Scholars Research Fund (2016SCU04A04).

\section{Author contributions}

All authors contributed toward data analysis, drafting and revising the paper and agree to be accountable for all aspects of the work.

\section{Disclosure}

The authors report no conflicts of interest in this work.

\section{References}

1. Chen L, Zhang W, Yan W, et al. The putative tumor suppressor miR524-5p directly targets Jagged-1 and Hes-1 in glioma. Carcinogenesis. 2012;33(11):2276-2282.

2. Haussecker D, Kay MA. RNA interference. Drugging RNAi. Science. 2015;347(6226):1069-1070.

3. Cambon K, Déglon N. Lentiviral-mediated gene transfer of siRNAs for the treatment of Huntington's disease. Methods Mol Biol. 2013; 1010:95-109.

4. Tseng YC, Mozumdar S, Huang L. Lipid-based systemic delivery of siRNA. Adv Drug Deliv Rev. 2009;61(9):721-731.

5. Whitehead KA, Langer R, Anderson DG. Knocking down barriers: advances in siRNA delivery. Nat Rev Drug Discov. 2009;8(2): 129-138.

6. Liu X, Wang X, Du W, et al. Suppressor of fused (Sufu) represses Gli1 transcription and nuclear accumulation, inhibits glioma cell proliferation, invasion and vasculogenic mimicry, improving glioma chemosensitivity and prognosis. Oncotarget. 2014;5(22):11681-11694.

7. Du WZ, Feng Y, Wang XF, et al. Curcumin suppresses malignant glioma cells growth and induces apoptosis by inhibition of SHH/GLI1 signaling pathway in vitro and vivo. CNS Neurosci Ther. 2013;19(12): 926-936.

8. Branzei D, Foiani M. Regulation of DNA repair throughout the cell cycle. Nat Rev Mol Cell Biol. 2008;9(4):297-308.

9. Numa Y, Tsukazaki Y, Yamamoto M, Matsudaira T, Kawamoto K. [Cyclin protein expression on malignant glioma cells]. Hum Cell. 1998;11(1):21-26. Japanese.

10. Chen ZP, Mohr G. Induced ERCC2 expression Vis-à-Vis BCNU resistance in human glioma cell lines. Chinese J Neurosurg. 2003;19(1): $18-21$.

11. Lytle RA, Jiang Z, Zheng X, Rich KM. BCNU down-regulates anti-apoptotic proteins $\mathrm{Bcl}-\mathrm{xL}$ and $\mathrm{Bcl}-2$ in association with cell death in oligodendroglioma-derived cells. J Neurooncol. 2004;68(3):233-241.

12. Jacob J, Briscoe J. Gli proteins and the control of spinal-cord patterning. EMBO Rep. 2003;4(8):761-765. 
13. Carpenter RL, Paw I, Hu Z, et al. The gain-of-function GLI1 transcription factor TGLI1 enhances expression of VEGF-C and TEM7 to promote glioblastoma angiogenesis. Oncotarget. 2015;6(26):22653-22665.

14. Lampichler K, Ferrer P, Vila G, et al. The role of proto-oncogene GLI1 in pituitary adenoma formation and cell survival regulation. Endocr Relat Cancer. 2015;22(5):793-803.

15. Singh RR, Kunkalla K, Qu C, et al. ABCG2 is a direct transcriptional target of hedgehog signaling and involved in stroma-induced drug tolerance in diffuse large B-cell lymphoma. Oncogene. 2011;30(49): 4874-4886.

16. Liu S, Duan X, Xu L, et al. Nuclear Glil expression is associated with pathological complete response and event-free survival in HER2positive breast cancer treated with trastuzumab-based neoadjuvant therapy. Tumour Biol. 2016;37(4):4873-4881.

17. Lee JM, Yoon TJ, Cho YS. Recent developments in nanoparticlebased siRNA delivery for cancer therapy. Biomed Res Int. 2013;2013: 782041.

18. Yuan X, Naguib S, Wu Z. Recent advances of siRNA delivery by nanoparticles. Expert Opin Drug Deliv. 2011;8(4):521-536.

19. Burnett JC, Rossi JJ, Tiemann K. Current progress of siRNA/shRNA therapeutics in clinical trials. Biotechnol J. 2011;6(9):1130-1146.
20. Liu YP, Berkhout B. miRNA cassettes in viral vectors: problems and solutions. Biochim Biophys Acta. 2011;1809(11-12):732-745.

21. Sinn PL, Sauter SL, McCray PB Jr. Gene therapy progress and prospects: development of improved lentiviral and retroviral vectors - design, biosafety, and production. Gene Ther. 2005;12(14):1089-1098.

22. Parvani JG, Jackson MW. Silencing the roadblocks to effective triplenegative breast cancer treatments by siRNA nanoparticles. Endocr Relat Cancer. 2017;24(4):R81-R97.

23. Stecca B, Ruiz i Altaba A. A GLI1-p53 inhibitory loop controls neural stem cell and tumor cell numbers. EMBO J. 2009;28(6):663-676.

24. Wang G, Zhang Z, Xu Z, et al. Activation of the sonic hedgehog signaling controls human pulmonary arterial smooth muscle cell proliferation in response to hypoxia. Biochim Biophys Acta. 2010;1803(12):1359-1367.

25. Fiaschi M, Rozell B, Bergström A, Toftgård R. Development of mammary tumors by conditional expression of GLI1. Cancer Res. 2009;69(11): 4810-4817.

26. Thomas ZI, Gibson W, Sexton JZ, et al. Targeting GLI1 expression in human inflammatory breast cancer cells enhances apoptosis and attenuates migration. Br J Cancer. 2011;104(10):1575-1586.
International Journal of Nanomedicine

\section{Publish your work in this journal}

The International Journal of Nanomedicine is an international, peerreviewed journal focusing on the application of nanotechnology in diagnostics, therapeutics, and drug delivery systems throughou the biomedical field. This journal is indexed on PubMed Central,

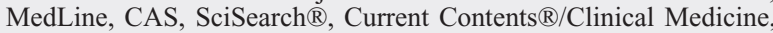

\section{Dovepress}

Journal Citation Reports/Science Edition, EMBase, Scopus and the Elsevier Bibliographic databases. The manuscript management system is completely online and includes a very quick and fair peer-review system, which is all easy to use. Visit http://www.dovepress.com/ testimonials.php to read real quotes from published authors. 